\title{
Temperature Effect on the Viscosity of Commercial
}

\section{Carrot Puree}

\author{
Virues-Delgadillo Jorge-Octavio, Domínguez-Rendón Ana-Cristina, López-del-Castillo-Lozano Micloth, \\ Domínguez-Cañedo Irma-Liliana and Galán-Méndez Frixia \\ Facultad de Ciencias Químicas, Universidad Veracruzana, Lomas del Estadio S/N, Xalapa, Veracruz 91000, México
}

\begin{abstract}
Temperature effect was evaluated over the viscosity of commercial carrot puree in the range 30 to $60{ }^{\circ} \mathrm{C}$, using a Brookfield viscometer, model 4535, Lab-Line instruments. In order to perform the viscosity tests, 28 specimens of carrot puree from a local market were used. Totally, 72 tests (each one with a couple replicas) were performed during five minutes, using four temperatures (30, 40, 50 and $60{ }^{\circ} \mathrm{C}$ ), six shear rates (0.26, 0.52, 1.05, 2.09, 5.24 and $\left.10.47 \mathrm{~s}^{-1}\right)$ and three spindles (4, 5 and 6). Statistical analysis was performed using LSD and Duncan tests with a confidence interval of 95\% ( $p$ value of 0.05 ). In the current study, it was observed that an increase in the temperature triggers a reduction in the viscosity of the carrot specimens analyzed, although the samples tested at $60{ }^{\circ} \mathrm{C}$ were deviated from this trend, which may be due to denaturation of the starch containing carrot samples at such temperature. Multiple biochemical and micro-structural changes inside the food specimens may begin to occur below and over the physiological range $\left(20-45^{\circ} \mathrm{C}\right)$. Experimental data were analyzed with Power Law model in order to obtain the best fitted parameters as a function of shear rate: It was observed that the consistent index $(\mathrm{k})$ decreased with an increment in the temperature; while all the values of parameter " $n$ " lesser than one, which means that the puree samples behaved as a pseudo-plastic fluid. Besides that, an Arrhenius model was used to fit the parameters as a function of temperature: Parameter A was lower using the experimental data at shear rates of $10.47 \mathrm{~s}^{-1}$. The modeling performed may be helpful for the appropriate selection of some process variables, as the shear rate and operation temperature, used for the production of carrot puree; all this is in order to obtain the finished product with the desired consistency.
\end{abstract}

Key words: Food rheology, viscosity, temperature, carrot puree.

\section{Introduction}

Carrots are a very popular vegetable in these days and important in the human diet, as it is a source of vitamins $\mathrm{A}, \mathrm{B}$ and $\mathrm{C}$, as well as carotenoids $[1,2]$. Carrot cultivation has experienced significant growth in recent years. In Mexico, the main government state producers of carrots are Guanajuato, Puebla and Zacatecas [3]. The rheological properties are essential for the proper design of equipment to produce various foods, such as juices, baby's puree, creams, jams, soups, etc.; and to determine its quality standards [4, 5]. The study of rheological behavior of liquid and semi-liquid food is complex, because most of them

Corresponding author: Virues-Delgadillo Jorge Octavio, $\mathrm{PhD}$ in chemical \& biological engineering, full time professor, research fields: food rheology and biomaterials mechanical testing. behave as non-Newtonian fluids; and therefore, it required appropriate instrumentation, such as a rotational viscometer. Furthermore, it is well known that the viscosity of most liquids depends heavily on temperature. Therefore, it is essential to determine the viscosity changes undergone by a food product into a mixer when it raises or lowers the processing temperature [6-9]. When querying scientific articles on the rheological properties of purees, it was not found publications that analyze the effect of temperature on the viscosity of carrot puree. As far as we know, it has only been analyzed the effect of solids concentration on samples of reconstituted carrot puree [10]. Therefore, the motivation to perform this study is justified.

\section{Methodology}

In order to perform viscosity tests, it was used 
commercial carrot puree (28 specimens) acquired in the local supermarket, a Brookfield type viscometer, an electric grill, a beaker of $600 \mathrm{~mL}$, spindles number 4 to 6 (Fig. 1), a stopwatch, a thermometer, and a bath to control the temperature. Diameter of Brookfield spindles is shown in Table 1 . The shear rate range used was from 0.26 to $10.47 \mathrm{~s}^{-1}$. For each selected temperature (30, 40, 50 and $60^{\circ} \mathrm{C}$ ), the viscosities of seven specimens were evaluated for 5 minutes using all possible combinations of the spindle and shear rate. These data were subjected to ANOVA and are expressed as the mean of the viscosities obtained. Statistical analysis was performed using the Student T-test. The level of significance was $p<0.05$. Data obtained at times of 240 and 300 seconds were adjusted to model the power law and Arrhenius equation, in order to find the parameters that best represent the behavior of carrot puree. The advantage of using these two mathematical equations is their wide application into a large number of non-Newtonian fluids and their relatively simple mathematical treatment. Statistical ANOVA tests of viscosity experimental carrot puree data were performed using SPSS 16.0 software, where Duncan subgroups (based on temperature, spindle, and shear rate) are shown in Table 4.

\section{Results}

In this work the effect of temperature on the viscosity of commercial carrot puree samples at different shear rates was analyzed using several spindles, as shown in Figs. 2 and 3. Regardless of the spindle and shear rate selected, an increase in temperature caused a decrease in the viscosity of the samples. However, the viscosity data at $60^{\circ} \mathrm{C}$ did not have the same tendency, mainly because their behaviors were similar to those corresponding to tests performed at $30^{\circ} \mathrm{C}$. It is likely that at $60^{\circ} \mathrm{C}$, the starch that must be inside carrot puree begins to denature and thereby increases the viscosity of the suspension.
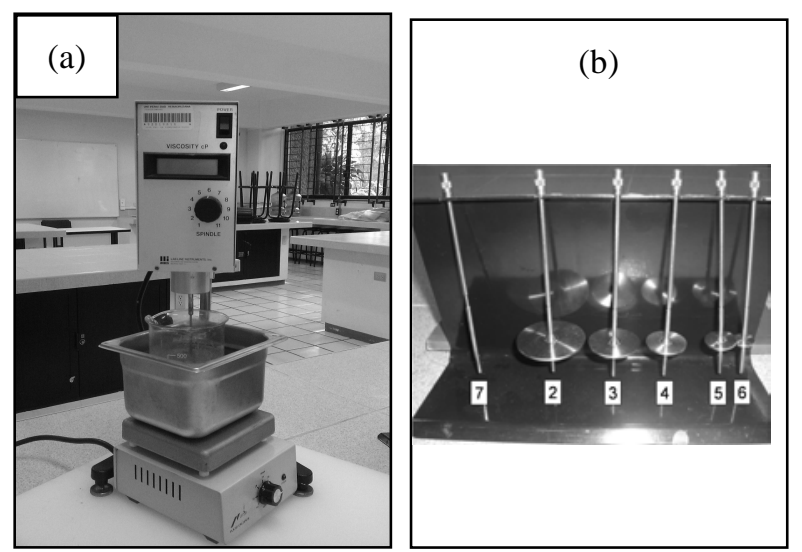

Fig. 1 (a) Typical carrot puree specimen analyzed on Brookfield Viscometer. (b) Brookfield Spindle numbers and geometry.

Table 1 Diameter of Brookfield Spindles.

\begin{tabular}{ll}
\hline Spindle Nr. & Diameter $(\mathrm{cm})$ \\
\hline 2 & 4.7 \\
3 & 3.5 \\
4 & 2.7 \\
5 & 2.1 \\
6 & 1.5 \\
7 & 0.2 \\
\hline
\end{tabular}

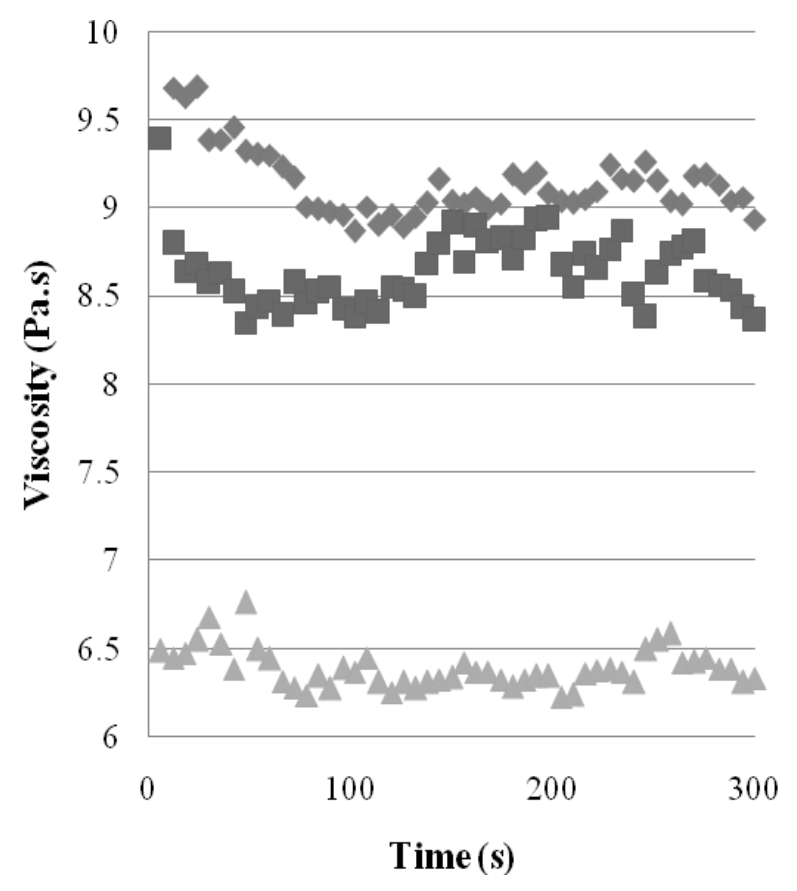

Fig. 2 Temperature effect over the viscosity of carrot puree typical simple using spindle $\mathrm{Nr} .4$ and shear rate of $1.05 \mathrm{rad} / \mathrm{s}$ : Diamonds $\left(30{ }^{\circ} \mathrm{C}\right)$, Squares $\left(40{ }^{\circ} \mathrm{C}\right)$ and Triangles $\left(50{ }^{\circ} \mathrm{C}\right)$. 


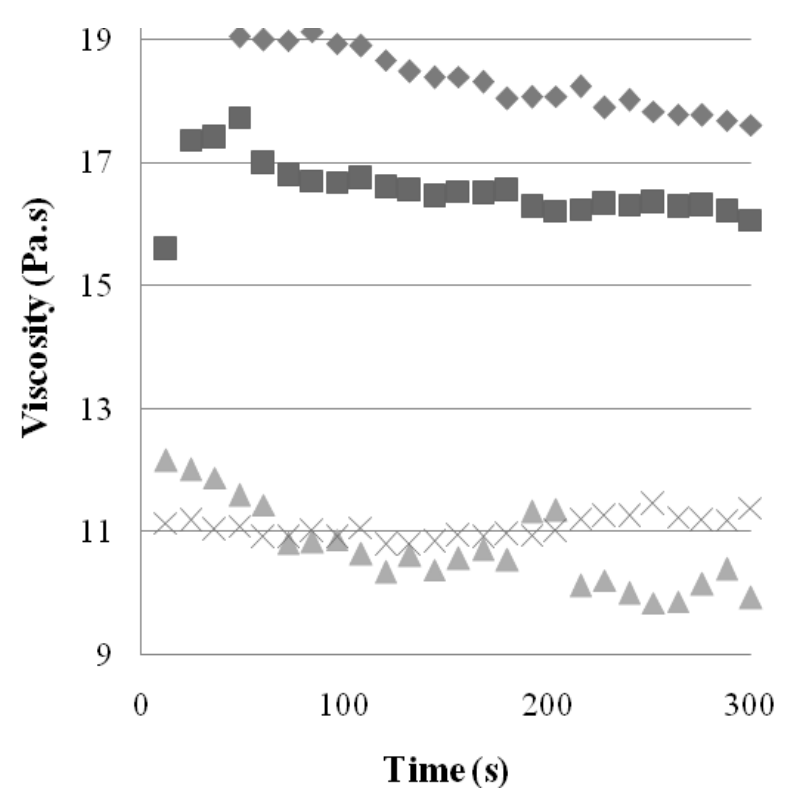

Fig. 3 Temperature effect over the viscosity of carrot puree typical simple using spindle $\mathrm{Nr} .5$ and shear rate of $0.52 \mathrm{rad} / \mathrm{s}$ : Diamonds $\left(30^{\circ} \mathrm{C}\right)$, Squares $\left(40{ }^{\circ} \mathrm{C}\right)$, Triangles $\left(50{ }^{\circ} \mathrm{C}\right)$, Crosses $\left(60^{\circ} \mathrm{C}\right)$.

It was possible to determine $n$ and $k$ (consistency index) parameters of puree samples analyzed, when power law model was applied to stress-shear rate curve data, as shown in Figs. 4 and 5. Power Law fitted parameters are reported in Table 2. It was observed a decrement in the values of the consistency index (k) with an increment of temperature; for example, this parameter was smaller for data at $60^{\circ} \mathrm{C}$ in comparison to the data obtained at the other testing temperatures. Therefore by increasing the value of $k$, sample viscosity increases. On the other hand, the parameter $n$ indicates how far away is the behavior of carrot puree samples from a Newtonian fluid $(n=1)$. All values of $n$ found in this work are less than unity, which indicates that carrot puree must have a behavior similar to a pseudoplastic fluid. With respect to the estimation of parameter A of Arrhenius law (Figs. 6 and 7), it was found that this parameter was lower than data obtained at shear rates closer to $10.47 \mathrm{~s}^{-1}$. Arrhenius fitted parameters are shown in Table 3. When the yield stress was calculated, it was also observed that the yield stress decreases its value with an increase in temperature. The effect of temperature, spindle, and shear rate is shown in Table 4, where Duncan statistical analysis was performed for all experimental specimens tested.

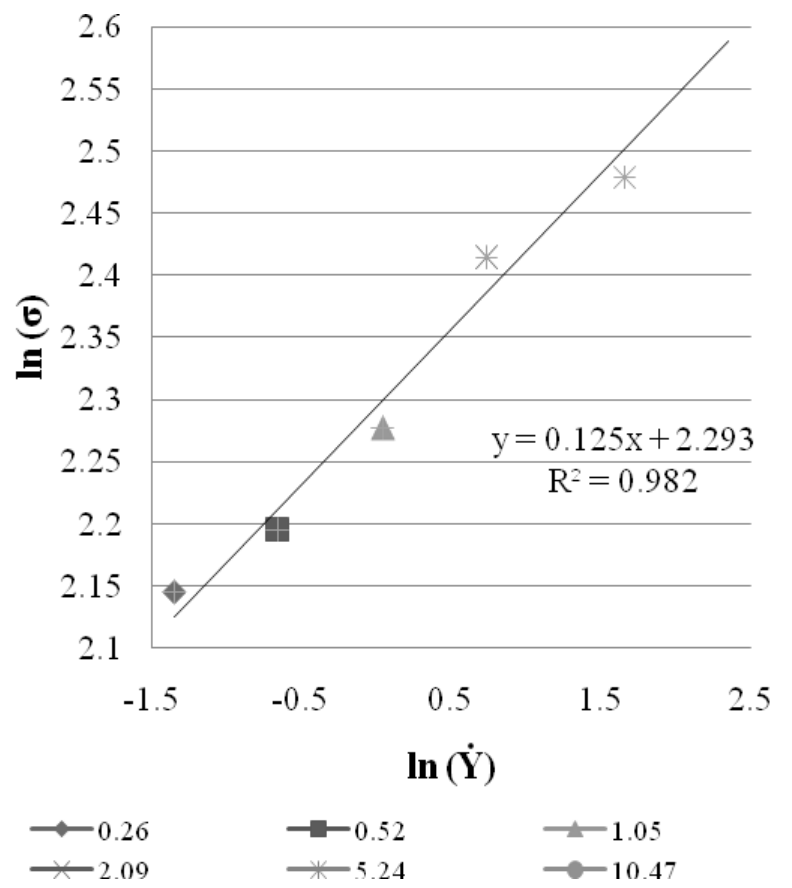

Fig. 4 Power Law fitted procedure at $60{ }^{\circ} \mathrm{C}$ using spindle Nr. 4.

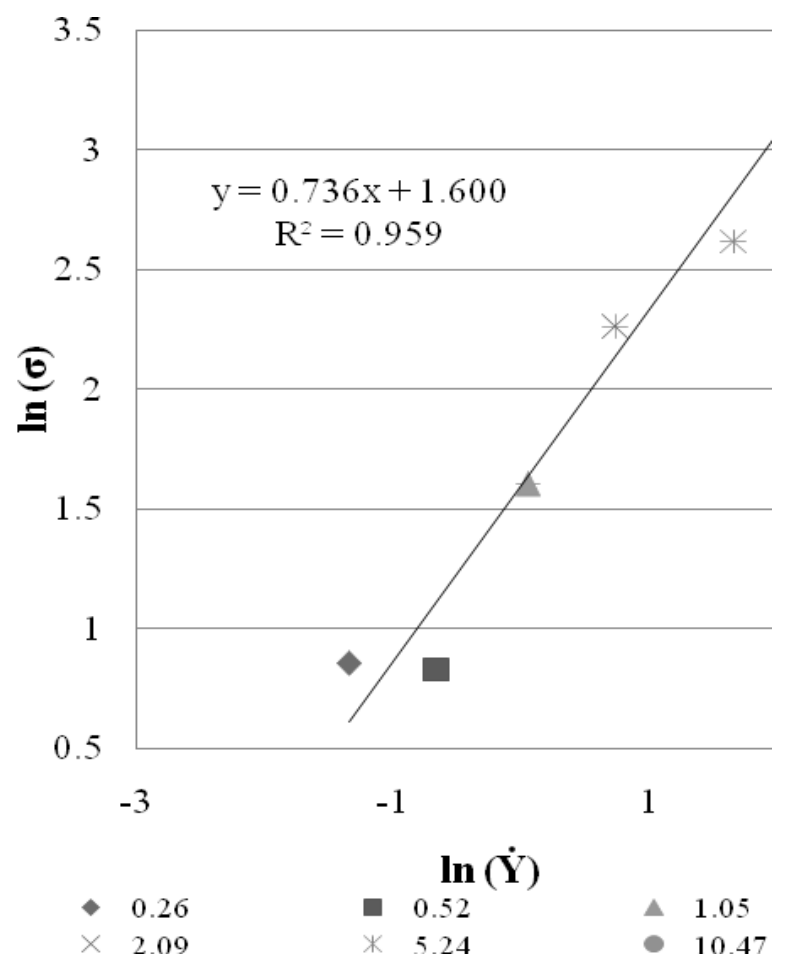

Fig. 5 Power Law fitted procedure at $60{ }^{\circ} \mathrm{C}$ using spindle Nr. 6. 
Table 2 Power Law fitted parameters.

\begin{tabular}{llll}
\hline Temperature $\left({ }^{\circ} \mathrm{C}\right)$ & Spindle Nr. & $k\left(\mathrm{~Pa}^{*} \mathrm{~s}\right)$ & $n$ \\
\hline \multirow{3}{*}{30} & 4 & 9.367 & 0.117 \\
& 5 & 9.450 & 0.148 \\
& 6 & 10.338 & 0.427 \\
\hline \multirow{3}{*}{40} & 4 & 9.488 & 0.104 \\
& 5 & 9.385 & 0.201 \\
& 6 & 17.949 & 0.215 \\
\hline \multirow{3}{*}{50} & 4 & 6.844 & 0.122 \\
& 5 & 6.233 & 0.226 \\
& 6 & 10.01 & 0.364 \\
60 & 4 & 9.792 & 0.128 \\
& 5 & 6.968 & 0.255 \\
& 6 & 5.017 & 0.730 \\
\hline
\end{tabular}

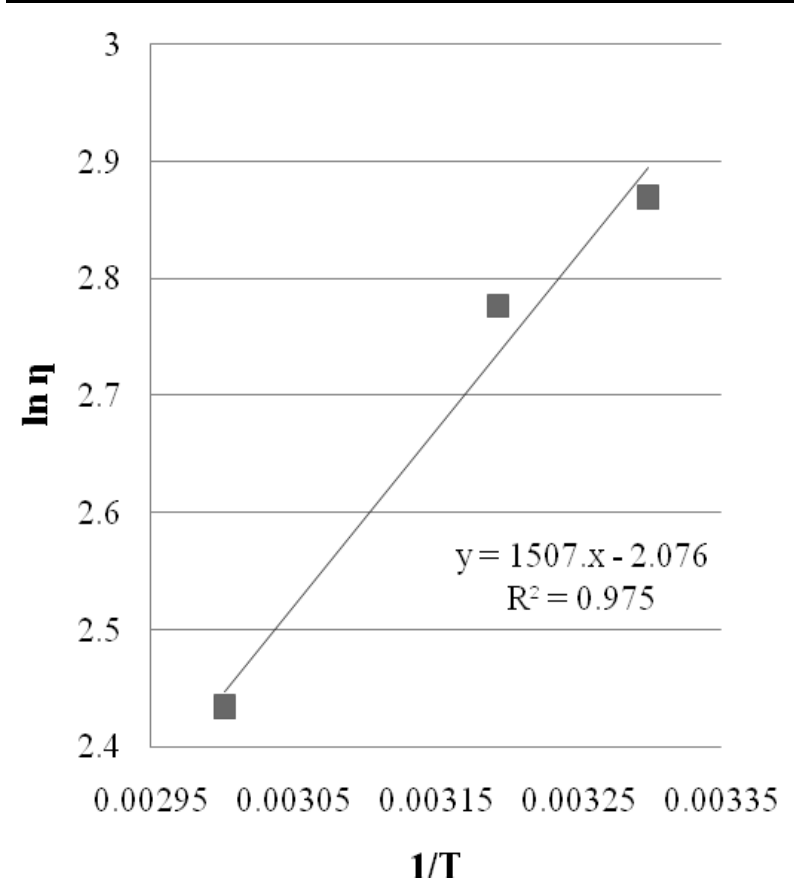

Fig. 6 Arrhenius equation fitted procedure at $0.52 \mathrm{rad} / \mathrm{s}$ using spindle Nr. 5.

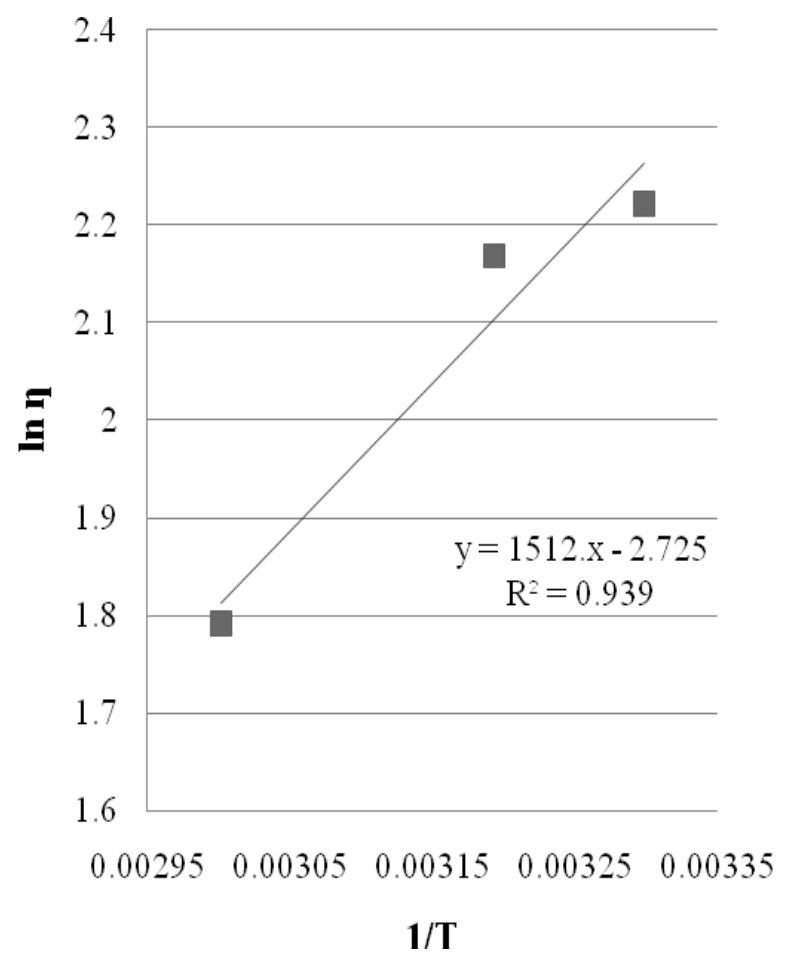

Fig. 7 Arrhenius equation fitted procedure at $1.05 \mathrm{rad} / \mathrm{s}$ using spindle Nr. 5 .

Table 3 Arrhenius Equation fitted parameters.

\begin{tabular}{llll}
\hline Shear rate $(1 / \mathrm{s})$ & Spindle Nr. & A $(\mathrm{Pa} * \mathrm{~s})$ & $\mathrm{E}(\mathrm{J} / \mathrm{mol})$ \\
\hline \multirow{2}{*}{0.26} & 4 & 4.987 & $-5,111.322$ \\
& 5 & 0.395 & $-11,261.313$ \\
\hline \multirow{2}{*}{0.52} & 4 & 18.988 & -277.904 \\
& 5 & 0.104 & $-13,032.195$ \\
\hline \multirow{2}{*}{1.05} & 4 & 16.327 & $-1,577.943$ \\
& 5 & 0.075 & $-13,053.396$ \\
\hline
\end{tabular}

Table 4 Duncan ANOVA results.

\begin{tabular}{llll}
\hline Group & Combination & $\begin{array}{l}\text { Mean } \\
\text { viscosity } \\
\text { (cP) }\end{array}$ & $p$-Value \\
\hline 1 & & $51,739.80$ & 1.000 \\
2 & T-40, Spindle 6, Shear 0.26 & $37,467.14$ & 1.000 \\
3 & T-40, Spindle 4, Shear 0.26 & $36,175.75$ & 1.000 \\
4 & T-30, Spindle 4, Shear 0.26 & $34,607.67$ & 0.102 \\
5 & T-60, Spindle 4, Shear 0.26 & $33,811.43$ & 0.078 \\
& T-40, Spindle 6, Shear 0.52 & $33,811.43$ & 0.181 \\
7 & T-40, Spindle 6, Shear 0.52 & $32,952.24$ & 0.175 \\
& T-40, Spindle 5, Shear 0.26 & $27,781.84$ & $27,130.23$ \\
\end{tabular}


(Table 4 continued)

\begin{tabular}{|c|c|c|c|}
\hline Group & Combination & $\begin{array}{l}\text { Mean } \\
\text { viscosity } \\
(\mathrm{cP}) \\
\end{array}$ & $\begin{array}{l}p \\
\text { value }\end{array}$ \\
\hline \multirow{2}{*}{9} & T-60, Spindle 4, Shear 0.52 & $17,759.60$ & \multirow{2}{*}{0.863} \\
\hline & T-30, Spindle 4, Shear 0.52 & $17,675.50$ & \\
\hline \multirow{2}{*}{10} & T-40, Spindle 5, Shear 0.52 & $16,548.57$ & \multirow{2}{*}{0.974} \\
\hline & T-40, Spindle 4, Shear 0.52 & $16,532.86$ & \\
\hline \multirow{2}{*}{11} & T-40, Spindle 6, Shear 1.05 & $15,529.71$ & \multirow{2}{*}{0.262} \\
\hline & T-50, Spindle 6, Shear 0.52 & $14,983.33$ & \\
\hline \multirow[t]{3}{*}{12} & T-50, Spindle 4, Shear 0.52 & $12,767.60$ & \multirow[t]{3}{*}{1.000} \\
\hline & T-30, Spindle 5, Shear 0.52 & $11,327.12$ & \\
\hline & T-30, Spindle 6, Shear 0.26 & $11,296.00$ & \\
\hline \multirow[t]{5}{*}{13} & T-60, Spindle 5, Shear 0.52 & $11,066.97$ & \multirow[t]{5}{*}{0.195} \\
\hline & T-50, Spindle 5, Shear 0.52 & $10,748.50$ & \\
\hline & T-60, Spindle 6, Shear 0.26 & $10,609.39$ & \\
\hline & T-60, Spindle 5, Shear 0.52 & $11,066.97$ & \\
\hline & T-50, Spindle 5, Shear 0.52 & $10,748.50$ & \\
\hline \multirow[t]{4}{*}{14} & T-60, Spindle 6, Shear 0.26 & $10,609.39$ & \multirow[t]{4}{*}{0.054} \\
\hline & T-30, Spindle 4, Shear 1.05 & $10,126.40$ & \\
\hline & T-50, Spindle 6, Shear 1.05 & $10,018.43$ & \\
\hline & T-30, Spindle 4, Shear 1.05 & $10,126.40$ & \\
\hline \multirow[t]{3}{*}{15} & T-50, Spindle 6, Shear 1.05 & $10,018.43$ & \multirow[t]{3}{*}{0.262} \\
\hline & T-60, Spindle 4, Shear 1.05 & $9,542.57$ & \\
\hline & T-60, Spindle 4, Shear 1.05 & $9,542.57$ & \\
\hline \multirow[t]{3}{*}{16} & T-40, Spindle 5, Shear 1.05 & $8,862.31$ & \multirow[t]{3}{*}{0.079} \\
\hline & T-40, Spindle 4, Shear 1.05 & $8,637.86$ & \\
\hline & T-40, Spindle 5, Shear 1.05 & $8,862.31$ & \\
\hline \multirow[t]{4}{*}{17} & T-40, Spindle 4, Shear 1.05 & $8,637.86$ & \multirow[t]{4}{*}{0.364} \\
\hline & T-40, Spindle 6, Shear 2.09 & $8,389.43$ & \\
\hline & T-50, Spindle 4, Shear 1.05 & $6,388.71$ & \\
\hline & T-60, Spindle 5, Shear 1.05 & $6,111.39$ & \\
\hline \multirow[t]{5}{*}{18} & T-30, Spindle 5, Shear 1.05 & $6,095.95$ & 0.102 \\
\hline & T-30, Spindle 6, Shear 1.05 & $5,614.12$ & \\
\hline & T-60, Spindle 4, Shear 2.09 & $5,490.54$ & \\
\hline & T-60, Spindle 5, Shear 1.05 & $6,111.39$ & \\
\hline & T-30, Spindle 5, Shear 1.05 & $6,095.95$ & \\
\hline 10 & T-30, Spindle 6, Shear 1.05 & $5,614.12$ & \\
\hline 19 & T-60, Spindle 4, Shear 2.09 & $5,490.54$ & 0.123 \\
\hline & T-50, Spindle 5, Shear 1.05 & $5,302.46$ & \\
\hline & T-30, Spindle 4, Shear 2.09 & $5,244.90$ & \\
\hline & T-30, Spindle 6, Shear 1.05 & $5,614.12$ & \\
\hline & T-60, Spindle 4, Shear 2.09 & $5,490.54$ & \\
\hline & T-50, Spindle 5, Shear 1.05 & $5,302.46$ & \\
\hline & T-30, Spindle 4, Shear 2.09 & $5,244.90$ & \\
\hline 20 & T-40, Spindle 6, Shear 5.24 & $4,843.29$ & 0.051 \\
\hline & T-50, Spindle 6, Shear 2.09 & $4,828.27$ & \\
\hline & T-60, Spindle 6, Shear 0.52 & $4,589.49$ & \\
\hline & T-40, Spindle 4, Shear 2.09 & $4,523.03$ & \\
\hline & T-40, Spindle 5, Shear 2.09 & $4,494.00$ & \\
\hline & T-60, Spindle 4, Shear 2.09 & $5,490.54$ & \\
\hline & T-50, Spindle 5, Shear 1.05 & $5,302.46$ & \\
\hline & T-30, Spindle 4, Shear 2.09 & $5,244.90$ & \\
\hline & T-40, Spindle 6, Shear 5.24 & $4,843.29$ & \\
\hline 21 & T-50, Spindle 6, Shear 2.09 & $4,828.27$ & 0.069 \\
\hline & T-60, Spindle 6, Shear 0.52 & $4,589.49$ & \\
\hline & T-40, Spindle 4, Shear 2.09 & $4,523.03$ & \\
\hline & T-40, Spindle 5, Shear 2.09 & $4,494.00$ & \\
\hline & T-30, Spindle 6, Shear 0.52 & $4,440.80$ & \\
\hline
\end{tabular}


(Table 4 continued)

\begin{tabular}{|c|c|c|c|}
\hline Group & Combination & $\begin{array}{l}\text { Mean } \\
\text { viscosity } \\
\text { (cP) }\end{array}$ & $\begin{array}{l}p \\
\text { value }\end{array}$ \\
\hline \multirow{9}{*}{22} & T-50, Spindle 5, Shear 1.05 & $5,302.46$ & \multirow{9}{*}{0.052} \\
\hline & T-30, Spindle 4, Shear 2.09 & $5,244.90$ & \\
\hline & T-40, Spindle 6, Shear 5.24 & $4,843.29$ & \\
\hline & T-50, Spindle 6, Shear 2.09 & $4,828.27$ & \\
\hline & T-60, Spindle 6, Shear 0.52 & $4,589.49$ & \\
\hline & T-40, Spindle 4, Shear 2.09 & $4,523.03$ & \\
\hline & T-40, Spindle 5, Shear 2.09 & $4,494.00$ & \\
\hline & T-30, Spindle 6, Shear 0.52 & $4,440.80$ & \\
\hline & T-30, Spindle 6, Shear 2.09 & $4,186.40$ & \\
\hline \multirow{8}{*}{23} & T-40, Spindle 6, Shear 5.24 & $4,843.29$ & \multirow{8}{*}{0.077} \\
\hline & T-50, Spindle 6, Shear 2.09 & $4,828.27$ & \\
\hline & T-60, Spindle 6, Shear 0.52 & $4,589.49$ & \\
\hline & T-40, Spindle 4, Shear 2.09 & $4,523.03$ & \\
\hline & T-40, Spindle 5, Shear 2.09 & $4,494.00$ & \\
\hline & T-30, Spindle 6, Shear 0.52 & $4,440.80$ & \\
\hline & T-30, Spindle 6, Shear 2.09 & $4,186.40$ & \\
\hline & T-60, Spindle 6, Shear 2.09 & $3,830.29$ & \\
\hline \multirow{7}{*}{24} & T-60, Spindle 6, Shear 0.52 & $4,589.49$ & \multirow{7}{*}{0.068} \\
\hline & T-40, Spindle 4, Shear 2.09 & $4,523.03$ & \\
\hline & T-40, Spindle 5, Shear 2.09 & $4,494.00$ & \\
\hline & T-30, Spindle 6, Shear 0.52 & $4,440.80$ & \\
\hline & T-30, Spindle 6, Shear 2.09 & $4,186.40$ & \\
\hline & T-60, Spindle 6, Shear 2.09 & $3,830.29$ & \\
\hline & T-60, Spindle 5, Shear 2.09 & $3,557.65$ & \\
\hline \multirow{8}{*}{25} & T-40, Spindle 4, Shear 2.09 & $4,523.03$ & \multirow{8}{*}{0.056} \\
\hline & T-40, Spindle 5, Shear 2.09 & $4,494.00$ & \\
\hline & T-30, Spindle 6, Shear 0.52 & $4,440.80$ & \\
\hline & T-30, Spindle 6, Shear 2.09 & $4,186.40$ & \\
\hline & T-60, Spindle 6, Shear 2.09 & $3,830.29$ & \\
\hline & T-60, Spindle 5, Shear 2.09 & $3,557.65$ & \\
\hline & T-50, Spindle 4, Shear 2.09 & $3,471.83$ & \\
\hline & T-30, Spindle 5, Shear 2.09 & $3,434.71$ & \\
\hline \multirow{8}{*}{26} & T-30, Spindle 6, Shear 2.09 & $4,186.40$ & \multirow{8}{*}{0.064} \\
\hline & T-60, Spindle 6, Shear 2.09 & $3,830.29$ & \\
\hline & T-60, Spindle 5, Shear 2.09 & $3,557.65$ & \\
\hline & T-50, Spindle 4, Shear 2.09 & $3,471.83$ & \\
\hline & T-30, Spindle 5, Shear 2.09 & $3,434.71$ & \\
\hline & T-60, Spindle 6, Shear 1.05 & $3,315.71$ & \\
\hline & T-40, Spindle 6, Shear 10.47 & $3,165.23$ & \\
\hline & T-60, Spindle 6, Shear 10.47 & $3,128.00$ & \\
\hline \multirow{9}{*}{27} & T-60, Spindle 6, Shear 2.09 & $3,830.29$ & \multirow{9}{*}{0.058} \\
\hline & T-60, Spindle 5, Shear 2.09 & $3,557.65$ & \\
\hline & T-50, Spindle 4, Shear 2.09 & $3,471.83$ & \\
\hline & T-30, Spindle 5, Shear 2.09 & $3,434.71$ & \\
\hline & T-60, Spindle 6, Shear 1.05 & $3,315.71$ & \\
\hline & T-40, Spindle 6, Shear 10.47 & $3,165.23$ & \\
\hline & T-60, Spindle 6, Shear 10.47 & $3,128.00$ & \\
\hline & T-50, Spindle 5, Shear 2.09 & $2,939.40$ & \\
\hline & T-60, Spindle 6, Shear 5.24 & $2,739.66$ & \\
\hline \multirow{7}{*}{28} & T-30, Spindle 5, Shear 2.09 & $3,434.71$ & \multirow{7}{*}{0.056} \\
\hline & T-60, Spindle 6, Shear 1.05 & $3,315.71$ & \\
\hline & T-40, Spindle 6, Shear 10.47 & $3,165.23$ & \\
\hline & T-60, Spindle 6, Shear 10.47 & $3,128.00$ & \\
\hline & T-50, Spindle 5, Shear 2.09 & $2,939.40$ & \\
\hline & T-60, Spindle 6, Shear 5.24 & $2,739.66$ & \\
\hline & T-30, Spindle 4, Shear 5.24 & $2,357.54$ & \\
\hline
\end{tabular}


(Table 4 continued)

\begin{tabular}{|c|c|c|c|}
\hline Group & Combination & $\begin{array}{l}\text { Mean } \\
\text { viscosity } \\
\text { (cP) }\end{array}$ & $\begin{array}{l}p \\
\text { value }\end{array}$ \\
\hline \multirow{9}{*}{29} & T-60, Spindle 6, Shear 1.05 & $3,315.71$ & \multirow{9}{*}{0.066} \\
\hline & T-40, Spindle 6, Shear 10.47 & $3,165.23$ & \\
\hline & T-60, Spindle 6, Shear 10.47 & $3,128.00$ & \\
\hline & T-50, Spindle 5, Shear 2.09 & $2,939.40$ & \\
\hline & T-60, Spindle 6, Shear 5.24 & $2,739.66$ & \\
\hline & T-30, Spindle 4, Shear 5.24 & $2,357.54$ & \\
\hline & T-60, Spindle 4, Shear 5.24 & $2,289.40$ & \\
\hline & T-40, Spindle 5, Shear 5.24 & $2,263.85$ & \\
\hline & T-40, Spindle 6, Shear 10.47 & $3,165.23$ & \\
\hline \multirow{6}{*}{30} & T-60, Spindle 6, Shear 10.47 & $3,128.00$ & \multirow{7}{*}{0.053} \\
\hline & T-50, Spindle 5, Shear 2.09 & $2,939.40$ & \\
\hline & T-60, Spindle 6, Shear 5.24 & $2,739.66$ & \\
\hline & T-30, Spindle 4, Shear 5.24 & $2,357.54$ & \\
\hline & T-60, Spindle 4, Shear 5.24 & $2,289.40$ & \\
\hline & T-40, Spindle 5, Shear 5.24 & $2,263.85$ & \\
\hline \multirow{9}{*}{31} & T-60, Spindle 5, Shear 5.24 & $2,065.00$ & \\
\hline & T-50, Spindle 5, Shear 2.09 & $2,939.40$ & \multirow{9}{*}{0.060} \\
\hline & T-60, Spindle 6, Shear 5.24 & $2,739.66$ & \\
\hline & T-30, Spindle 4, Shear 5.24 & $2,357.54$ & \\
\hline & T-60, Spindle 4, Shear 5.24 & $2,289.40$ & \\
\hline & T-40, Spindle 5, Shear 5.24 & $2,263.85$ & \\
\hline & T-60, Spindle 5, Shear 5.24 & $2,065.00$ & \\
\hline & T-30, Spindle 5, Shear 5.24 & $1,993.57$ & \\
\hline & T-40, Spindle 4, Shear 5.24 & $1,866.64$ & \\
\hline & T-60, Spindle 6, Shear 5.24 & $2,739.66$ & \\
\hline \multirow{6}{*}{32} & T-30, Spindle 4, Shear 5.24 & $2,357.54$ & \multirow{7}{*}{0.106} \\
\hline & T-60, Spindle 4, Shear 5.24 & $2,289.40$ & \\
\hline & T-40, Spindle 5, Shear 5.24 & $2,263.85$ & \\
\hline & T-60, Spindle 5, Shear 5.24 & $2,065.00$ & \\
\hline & T-30, Spindle 5, Shear 5.24 & $1,993.57$ & \\
\hline & T-40, Spindle 4, Shear 5.24 & $1,866.64$ & \\
\hline \multirow{16}{*}{33} & T-50, Spindle 5, Shear 5.24 & $1,809.10$ & \\
\hline & T-30, Spindle 4, Shear 5.24 & $2,357.54$ & \multirow{15}{*}{0.065} \\
\hline & T-60, Spindle 4, Shear 5.24 & $2,289.40$ & \\
\hline & T-40, Spindle 5, Shear 5.24 & $2,263.85$ & \\
\hline & T-60, Spindle 5, Shear 5.24 & $2,065.00$ & \\
\hline & T-30, Spindle 5, Shear 5.24 & $1,993.57$ & \\
\hline & T-40, Spindle 4, Shear 5.24 & $1,866.64$ & \\
\hline & T-50, Spindle 5, Shear 5.24 & $1,809.10$ & \\
\hline & T-40, Spindle 5, Shear 10.47 & $1,451.62$ & \\
\hline & T-40, Spindle 4, Shear 10.47 & $1,390.12$ & \\
\hline & T-60, Spindle 5, Shear 10.47 & $1,362.06$ & \\
\hline & T-60, Spindle 4, Shear 10.47 & $1,350.50$ & \\
\hline & T-50, Spindle 4, Shear 5.24 & $1,349.73$ & \\
\hline & T-30, Spindle 5, Shear 10.47 & $1,307.73$ & \\
\hline & T-30, Spindle 4, Shear 10.47 & $1,298.14$ & \\
\hline & T-50, Spindle 6, Shear 5.24 & $1,249.72$ & \\
\hline
\end{tabular}


(Table 4 continued)

\begin{tabular}{|c|c|c|c|}
\hline Group & Combination & $\begin{array}{l}\text { Mean } \\
\text { viscosity } \\
\text { (cP) }\end{array}$ & $\begin{array}{l}p \\
\text { value }\end{array}$ \\
\hline \multirow{14}{*}{34} & T-60, Spindle 4, Shear 5.24 & $2,289.40$ & \multirow{14}{*}{0.055} \\
\hline & T-40, Spindle 5, Shear 5.24 & $2,263.85$ & \\
\hline & T-60, Spindle 5, Shear 5.24 & $2,065.00$ & \\
\hline & T-30, Spindle 5, Shear 5.24 & $1,993.57$ & \\
\hline & T-40, Spindle 4, Shear 5.24 & $1,866.64$ & \\
\hline & T-50, Spindle 5, Shear 5.24 & $1,809.10$ & \\
\hline & T-40, Spindle 5, Shear 10.47 & $1,451.62$ & \\
\hline & T-40, Spindle 4, Shear 10.47 & $1,390.12$ & \\
\hline & T-60, Spindle 5, Shear 10.47 & $1,362.06$ & \\
\hline & T-60, Spindle 4, Shear 10.47 & $1,350.50$ & \\
\hline & T-50, Spindle 4, Shear 5.24 & $1,349.73$ & \\
\hline & T-30, Spindle 5, Shear 10.47 & $1,307.73$ & \\
\hline & T-30, Spindle 4, Shear 10.47 & $1,298.14$ & \\
\hline & T-50, Spindle 6, Shear 5.24 & $1,249.72$ & \\
\hline \multirow{16}{*}{35} & T-50, Spindle 5, Shear 10.47 & $1,139.62$ & \multirow{16}{*}{0.092} \\
\hline & T-60, Spindle 5, Shear 5.24 & $2,065.00$ & \\
\hline & T-30, Spindle 5, Shear 5.24 & $1,993.57$ & \\
\hline & T-40, Spindle 4, Shear 5.24 & $1,866.64$ & \\
\hline & T-50, Spindle 5, Shear 5.24 & $1,809.10$ & \\
\hline & T-40, Spindle 5, Shear 10.47 & $1,451.62$ & \\
\hline & T-40, Spindle 4, Shear 10.47 & $1,390.12$ & \\
\hline & T-60, Spindle 5, Shear 10.47 & $1,362.06$ & \\
\hline & T-60, Spindle 4, Shear 10.47 & $1,350.50$ & \\
\hline & T-50, Spindle 4, Shear 5.24 & $1,349.73$ & \\
\hline & T-30, Spindle 5, Shear 10.47 & $1,307.73$ & \\
\hline & T-30, Spindle 4, Shear 10.47 & $1,298.14$ & \\
\hline & T-50, Spindle 6, Shear 5.24 & $1,249.72$ & \\
\hline & T-50, Spindle 5, Shear 10.47 & $1,139.62$ & \\
\hline & T-50, Spindle 4, Shear 10.47 & $1,051.21$ & \\
\hline & T-40, Spindle 5, Shear 10.47 & $1,451.62$ & \\
\hline \multirow{10}{*}{36} & T-40, Spindle 4, Shear 10.47 & $1,390.12$ & \multirow{10}{*}{0.053} \\
\hline & T-60, Spindle 5, Shear 10.47 & $1,362.06$ & \\
\hline & T-60, Spindle 4, Shear 10.47 & $1,350.50$ & \\
\hline & T-50, Spindle 4, Shear 5.24 & $1,349.73$ & \\
\hline & T-30, Spindle 5, Shear 10.47 & $1,307.73$ & \\
\hline & T-30, Spindle 4, Shear 10.47 & $1,298.14$ & \\
\hline & T-50, Spindle 6, Shear 5.24 & $1,249.72$ & \\
\hline & T-50, Spindle 5, Shear 10.47 & $1,139.62$ & \\
\hline & T-50, Spindle 4, Shear 10.47 & $1,051.21$ & \\
\hline & T-30, Spindle 6, Shear 5.24 & 456.26 & \\
\hline \multirow{13}{*}{37} & T-30, Spindle 6, Shear 10.47 & 312.20 & \multirow{13}{*}{0.063} \\
\hline & T-40, Spindle 4, Shear 10.47 & $1,390.12$ & \\
\hline & T-60, Spindle 5, Shear 10.47 & $1,362.06$ & \\
\hline & T-60, Spindle 4, Shear 10.47 & $1,350.50$ & \\
\hline & T-50, Spindle 4, Shear 5.24 & $1,349.73$ & \\
\hline & T-30, Spindle 5, Shear 10.47 & $1,307.73$ & \\
\hline & T-30, Spindle 4, Shear 10.47 & $1,298.14$ & \\
\hline & T-50, Spindle 6, Shear 5.24 & $1,249.72$ & \\
\hline & T-50, Spindle 5, Shear 10.47 & $1,139.62$ & \\
\hline & T-50, Spindle 4, Shear 10.47 & $1,051.21$ & \\
\hline & T-30, Spindle 6, Shear 5.24 & 456.26 & \\
\hline & T-30, Spindle 6, Shear 10.47 & 312.20 & \\
\hline & T-50, Spindle 6, Shear 10.47 & 291.94 & \\
\hline
\end{tabular}




\section{Conclusions}

In this study it was found that an increase in temperature caused a decrease in the viscosity of carrot puree samples, although the samples tested at $60{ }^{\circ} \mathrm{C}$ deviate from this trend. The latter may be due to the possible denaturation of starch that contains carrot specimens. Multiple biochemical and micro-structural changes $[11,12]$ might start in food products above or below the physiological range $\left(20-45^{\circ} \mathrm{C}\right)$. In order to corroborate this theory, subsequent carrot puree microstructure studies are suggested. A total of 37 different groups were determined using Duncan statistical test, from nearly 72 possible combinations of temperature, spindle and shear rate used to obtain the viscosity of each specimen analyzed. By closer analysis of power model parameters $n$ and $k$, it was demonstrated that carrot puree samples behaved like a pseudoplastic fluid. Furthermore, yield stress for each temperature tested was determined using Arrhenius Law estimated parameters, which can aid in the selection of some design variables in the mixer, such as stirring rate and operation temperature. Those production variables might help to obtain the finished product with the desired consistency or viscosity.

\section{References}

[1] Duke, J. A. 1983. Handbook of Energy Crops. Pardue University.

[2] FAO. 2006. "Fichas Técnicas Productos Frescos y Procesados." Recuperado de http://www.fao.org/inpho_archive/content/documents/vli brary/ae620s/index.htm.
[3] SAGARPA. 2013. "Producción Anual de Zanahoria en México 2013.” SIAP, Recuperado de http://www.siap.gob.mx.

[4] Bayod, E., Bolmstedt, U., Innings, F., and Tornberg, E. 2005. "Rheological Characterization of Fiber Suspensions Prepared from Vegetable Pulp and Dried Fiber: A Comparative Study.” Annual Transactions of the Nordic Rheology Society 13: 249-53.

[5] Ramírez-Navas, J. S. 2006. Fundamentos de Reología de Alimentos. Calí Valle, Colombia, 10: 1-46.

[6] Chiralt-Boix, A., Martínez-Navarrete, N., Camacho-Vidal, M. M., and González-Martínez, C. 1998. Experimentos de Fisicoquímica de Alimentos. Valencia, Servicios de Publicaciones SPUPV, Reproval, S. L.

[7] Muller, H. G. 1973. Introduccion a la reología de los alimentos. Zaragoza, España. Editorial Acribia.

[8] Silva, D. C. S., Braga, A. C. C., Lourenço, L. F. H., Rodrigues, A. M., and Peixoto Joele, M. R. S. 2017. "Rheological Behavior of Mixed Nectars of Pineapple Skin Juice and Tropical Fruit Pulp.” International Food Research Journal 24 (4): 1713-20.

[9] Sharma, M., Kristo, E., Corredig, M., and Duizer, L. 2017."Effect of Hydrocolloid Type on Texture of Pureed Carrots: Rheological and Sensory Measures." Food Hydrocolloids 63: 478-87.

[10] Moelants, K. R. N., Cardinaels, R., Jolie, R. P., Verrijssen, T. A. J., Van Buggenhout, S., Zumalacarregui, L. M., Van Loey, A. M., Moldenaers, P., and Hendrickx, M. E. 2011. "Relation between Particle Properties and Rheological Characteristics of Carrot-Derived Suspensions.” Food Bioprocess Technol. 1-17.

[11] Alvarez, M. D., and Canet, W. 2001. "Kinetics of Termal Softening of Potato Tissue Heated by Different Methods.” Eur. Food Res Technol. 212: 454-64.

[12] González-Martínez, G. 2003. "Heat-Induced Cell Membrane Injury of Vegetable Tissues-An Applied Study on Potatoes.” PhD thesis, Food Engineering, Lund University. 